\title{
Sirtuin 2 expression levels may predict the progression of sepsis survivors to chronic critical illness
}

\author{
Hua $\mathrm{Xu}^{1}$, Jiafu $\mathrm{Li}^{2}$, Xin Yu ${ }^{2}$, Huihong Zhang ${ }^{1}$, Hongmei Gao ${ }^{1}$, Bing Wang ${ }^{1}$, Yongqiang Wang ${ }^{1}$ \\ ${ }^{1}$ Department of Intensive Care Unit, Key Laboratory for Critical Care Medicine of the Ministry of Health, Emergency Medicine Research Institute, \\ Tianjin First Center Hospital, School of Medicine, Nankai University, Tianjin, China; ${ }^{2}$ Department of Critical Care Medicine, The First Central \\ Clinical College of Tianjin Medical University, Tianjin, China \\ Contributions: (I) Conception and design: H Xu; (II) Administrative support: B Wang, Y Wang; (III) Provision of study materials or patients: H Xu, \\ B Wang, Y Wang; (IV) Collection and assembly of data: H Zhang, X Yu, J Li; (V) Data analysis and interpretation: H Xu, H Gao; (VI) Manuscript \\ writing: All authors; (VII) Final approval of manuscript: All authors. \\ Correspondence to: Yongqiang Wang; Bing Wang. Department of Intensive Care Unit, Key Laboratory for Critical Care Medicine of the Ministry of \\ Health, Emergency Medicine Research Institute, Tianjin First Center Hospital, School of Medicine, Nankai University, 24 Fukang Road, Tianjin \\ 300192, China. Email: yongqiangwang1962@sina.com; egenbing@163.com.
}

Background: Sirtuin 2 (SIRT2) is a conserved deacetylase that participates in the regulation of inflammation in sepsis. In this observational prospective study, we investigated the predictive value of the SIRT2 expression level in the development of chronic critical illness (CCI) in patients with sepsis.

Methods: A total of 128 critically ill patients with sepsis or septic shock were enrolled and assigned to the CCI group, rapid recovery (RAP) group, or early death group according to their clinical trajectories. Patients' demographic and clinical information, as well as laboratory data, including C-reactive protein (CRP) level and total lymphocyte counts, were collected. Blood samples were obtained at admission and on days 1, 4, 7, 10, 14, and 21 (days 14 and 21 for the CCI group only). Peripheral blood mononuclear cells were isolated, and SIRT2 expression was measured by real-time polymerase chain reaction. Serum levels of interleukin (IL)-6 and IL-10 were measured by enzyme-linked immunosorbent assay.

Results: Our cohort included 37 CCI and 82 RAP patients, and 9 early death patients, who died within 14 days of intensive care unit (ICU) admission. Compared with the RAP group, CCI patients showed elevated CRP and IL-6 levels throughout the observation period, reflecting a sustained inflammatory response. However, decreases in total lymphocyte count and IL-10 expression in these patients were indicative of immunosuppression. SIRT2 mRNA level was lower in CCI and RAP patients compared with healthy controls at the initial stage of hospitalization, but increased starting on day 4 and continued to increase for the duration of hospitalization. By day 10, SIRT2 expression had almost returned to normal in RAP patients; however, in CCI patients, it continued to increase until the end of the observation period. A receiver-operating characteristic curve analysis showed that the expression level of SIRT2 at 10 days predicts the occurrence of CCI $(\mathrm{P}<0.05)$.

Conclusions: SIRT2 expression may be a useful marker for identifying sepsis survivors who are at risk of progressing to CCI.

Keywords: Chronic critical illness (CCI); sirtuin 2 (SIRT2); rapid recovery; sepsis; septic shock

Submitted Nov 16, 2020. Accepted for publication Jan 02, 2021.

doi: 10.21037/atm-20-7975

View this article at: http://dx.doi.org/10.21037/atm-20-7975 


\section{Introduction}

Sepsis is defined as a life-threatening organ dysfunction caused by an impaired host response to infection, and is one of the leading causes of death in critically ill patients (1). In 2017, there were 48.9 million new cases of sepsis globally and 11 million sepsis-related deaths, accounting for $19.7 \%$ of all deaths (2). The high morbidity, mortality, and costs associated with sepsis place a burden on healthcare systems around the world. Advances in high-quality care and treatment strategies and timely interventions have resulted in reduced mortality rates in cases of acute sepsis $(3,4)$. However, with an increase in the number of surviving patients, there are more cases that progress to chronic critical illness (CCI), although the pathophysiological mechanisms involved remain unclear.

Sepsis patients in the intensive care unit (ICU) with systemic inflammatory response syndrome who are resuscitated typically follow 1 of 3 clinical trajectories as follows: (I) progression to multiple organ failure and early death, which occurs in a small number of patients; (II) rapid recovery (RAP) from organ dysfunction, which is experienced by nearly half of patients; and (III) development of CCI, which often has poor long-term outcomes $(5,6)$. The clinical manifestations of CCI-also known as persistent inflammation, immunosuppression, and catabolism syndrome (PICS) - include an abnormal immune response, which can undermine treatment efficacy. It is therefore important to clarify the role of immune dysfunction in the development of CCI in sepsis patients.

Sirtuin (SIRT) 2 is the only member of the SIRT family that is localized in the cytoplasm, but can translocate to the nucleus $(7,8)$. In recent years, multiple studies have shown that SIRT2 is involved in inflammation in sepsis. However, there have been no reports on SIRT2 expression levels in sepsis patients and there have only one report on the prediction of sepsis survivors to chronic critical illness (9). Our preliminary research showed that SIRT2 expression is lower in early-stage sepsis patients than in healthy individuals. Based on this observation, in the present study, we investigated the relationship between SIRT2 level and the development of CCI in patients with sepsis and septic shock who were admitted to the ICU.

We present the following article in accordance with the TRIPOD reporting checklist (available at http://dx.doi. org/10.21037/atm-20-7975).

\section{Methods}

\section{Study design and population}

This observational and prospective cohort study was approved by the Tianjin First Center Hospital Ethics Committee (approval No. $2018 \mathrm{~N} 085 \mathrm{KY}$ ), and is currently registered with Chinese Clinical Trial Registry (ChiCTR1800019038; http://www.chictr.org.cn/index. aspx). Clinical samples were collected from patients and were handled in accordance with the Declaration of Helsinki (as revise in 2013), and informed consent was obtained from patients or their legal representatives.

Between July 2018 and November 2019, 128 critically ill patients with sepsis or septic shock were enrolled within $24 \mathrm{~h}$ of admission to the ICU of Tianjin First Center Hospital. Patients were diagnosed according to The Third International Consensus Definitions for Sepsis and Septic Shock, which define sepsis as life-threatening organ dysfunction caused by a dysregulated host response to infection, and septic shock as a clinical construct of sepsis characterized by persisting hypotension requiring vasopressors to maintain mean arterial pressure (MAP) $\geq 65 \mathrm{mmHg}$ and a serum lactatelevel $>2 \mathrm{mmol} / \mathrm{L}$, despite adequate volume resuscitation (1).

Patients meeting any of the following criteria were excluded: (I) aged <18 years; (II) pregnant; (III) human immunodeficiency virus infection; (IV) autoimmune diseases or acquired immune deficiency syndrome; (V) underwent transplantation, chemotherapy, or radiotherapy in the 30 days before the onset of sepsis; (VI) severe traumatic brain injury; (VII) spinal cord injury resulting in permanent sensory and/or motor deficits; (VIII) heart failure; (IX) sepsis with an uncontrollable cause (e.g., unresectable bowel ischemia); (X) expected lifespan $<3$ months due to severe pre-existing comorbidities; and (XI) declined to provide consent.

Enrolled patients were grouped according to the abovementioned clinical trajectories. The CCI group comprised patients with an ICU stay $\geq 14$ days, with evidence of persistent organ dysfunction based on components of the Sequential Organ Failure Assessment (SOFA) score. The RAP group comprised patients who were discharged from the ICU within 14 days with a resolution of organ dysfunction. The early death group comprised patients who died within 14 days of admission to the ICU (5). In total, 37 CCI patients, 82 RAP patients, and 9 early death patients were ultimately enrolled.

We also recruited 20 healthy volunteers (12 males and 
8 females) from the Physical Examination Department of Tianjin First Center Hospital as the control group. The mean age of the controls was 44.6 years (range: $24-63$ years).

\section{Data collection}

The clinical characteristics of the patients, including sex, age, and source of infection, were recorded at admission; C-reactive protein (CRP) level and total lymphocyte count were evaluated at admission and on days 1, 4, 7, 10, 14, and 21 of hospitalization (days 14 and 21 in CCI group only). Laboratory examinations were performed at the same time points. The Acute Physiology and Chronic Health Evaluation (APACHE II) scores and SOFA scores of the patients were calculated based on the related clinical and demographic data.

\section{Blood sampling}

Peripheral blood samples were collected from patients at admission and on days 1, 4, 7, 10, 14, and 21 (days 14 and 21 in the CCI group only). Peripheral blood mononuclear cells (PBMCs) were isolated by Ficoll-Hypaque density gradient centrifugation and prepared for subsequent RNA extraction. Total RNA was extracted from $2 \times 10^{6}$ PBMCs using TRIzol reagent (Invitrogen, Carlsbad, CA, USA). Reverse transcription (RT) reaction was then carried out using SuperScript II RT (Invitrogen, USA) according to the manufacturer's protocol. Serum obtained from blood samples was stored as aliquots at $-80{ }^{\circ} \mathrm{C}$ until use. Interleukin (IL)-6 and IL-10 levels in serum were measured by enzyme linked immunosorbent assay (ELISA) using the Human IL-6 and Human IL-10 ELISA Kits (Solarbio, Beijing, China) according to the manufacturer's instructions.

\section{Real-time polymerase chain reaction (RT-PCR)}

The expression of SIRT2 in PBMCs of sepsis patients were evaluated by RT-PCR using the SYBR Green PCR kit (Takara Bio, Otsu, Japan) on an ABI PRISM-7500 Sequence Detection System (Applied Biosystems, Foster City, CA, USA). Primer sequences were as follows: SIRT2, 5'-ACGCTGTCGCAGAGTCAT-3' (forward) and 5'-CGCTCCAGGGTATCTATGTT-3' (reverse); and human GAPDH (internal control), 5'-GAAGGTGAAGGTCGGAGTC-3' (forward) and 5'-GAAGATGGTGATGGGATTTC-3' (reverse).

Reactions were performed in a total volume of $20 \mu \mathrm{L}$ containing $2 \mu \mathrm{L}$ of sample cDNA and $0.2 \mu \mathrm{M}$ of each primer. The thermal cycling conditions were $95^{\circ} \mathrm{C}$ for $30 \mathrm{~s}$, followed by 40 cycles of $95^{\circ} \mathrm{C}$ for $5 \mathrm{~s}$ and $60^{\circ} \mathrm{C}$ for $34 \mathrm{~s}$. SIRT2 transcription level was determined relative to that of GAPDH using the $2^{-\Delta \Delta \mathrm{Ct}} \operatorname{method}(\Delta \Delta \mathrm{Ct}=[\mathrm{CtSIRT2}-$ CtGAPDH $]$ sample - [CtSIRT2 - CtGAPDH $]$ calibrator).

\section{Statistical analysis}

Data were analyzed using SPSS version 19.0 software (IBM, Armonk, NY, USA) and R version 3.5.3 (https://www. r-project.org/). Normally distributed data are presented as mean \pm standard deviation, and were analyzed with the Student's t-test or by one-way analysis of variance. Data with non-normal distribution were analyzed with a nonparametric rank sum test. A mixed model was performed to analyze the differences between detection time points. Logistic regression analysis was performed for the variables, and receiver-operating characteristic curves were drawn using MedCalc software (Ostend, Belgium). The area under the curve (AUC) was calculated to assess the predictive utility of SIRT2 for the progression of sepsis or septic shock to CCI. $\mathrm{P}<0.05$ was considered statistically significant in all analyses.

\section{Results}

\section{Patient characteristics}

A total of 128 patients with sepsis or septic shock were enrolled in the present study. Of these patients, 37 developed CCI, 82 showed RAP, and 9 died within 14 days of ICU admission. The baseline demographic and clinicopathological information of the study cohort is summarized in Table 1. There were no differences between the CCI and RAP groups in terms of sex and age. The median age of the enrolled patients was 68 years. The incidence of septic shock was higher in the CCI group than in the RAP group (43.2\% vs. $35.4 \%$ ). CCI patients had a higher APACHE II score (21 vs. 16.5, $\mathrm{P}=0.0002)$ and SOFA score (9 vs. $6, \mathrm{P}=0.0007)$ than RAP patients. The CCI patients had a longer ICU stay than the RAP patients (20.1 vs. 8.2, $\mathrm{P}<0.0001)$. There was no difference in terms of source of infection. Mortality at 28 and 180 days was higher in the CCI group than in the RAP group ( $16.2 \%$ vs. $2.4 \%$ and $37.8 \%$ vs. $6.1 \%$, respectively).

\section{Laboratory findings in sepsis and septic shock patients}

In the present study, we found that the CRP level declined 
Table 1 Demographic and clinical characteristics of the study population

\begin{tabular}{|c|c|c|c|c|}
\hline Characteristics & $\mathrm{CCl}(\mathrm{n}=37)$ & RAP $(n=82)$ & Early death $(n=9)$ & $\mathrm{P}$ value $(\mathrm{CCl}$ vs. $\mathrm{RAP})$ \\
\hline Age (years, mean $\pm S D$ ) & $69.7 \pm 14.9$ & $67.7 \pm 14.4$ & $71 \pm 11.7$ & 0.4032 \\
\hline Age $\geq 65$ years, $n(\%)$ & $25(67.6)$ & $50(61.0)$ & $6(66.7)$ & \\
\hline APACHE II, median [25th, 75th] & $21[16,28]$ & $16.5[12,20]$ & $33[31,37]$ & 0.0002 \\
\hline \multicolumn{5}{|l|}{ Sepsis severity, n (\%) } \\
\hline Sepsis & $17(56.8 .9)$ & $53(64.6)$ & $2(22.2)$ & \\
\hline Septic shock & $16(43.2)$ & $29(35.4)$ & $7(77.8)$ & \\
\hline ICU LOS (mean \pm SD) & $20.1 \pm 5.3$ & $8.2 \pm 2.5$ & $9.2 \pm 2.8$ & $<0.001$ \\
\hline \multicolumn{5}{|l|}{ Source of infection, $\mathrm{n}(\%)$} \\
\hline Digestive system & $10(27)$ & $21(25.6)$ & $2(22.2)$ & \\
\hline Urinary tract & $4(10.8)$ & $7(8.5)$ & 0 & \\
\hline Intra-abdominal & $2(5.4)$ & $5(6.1)$ & 0 & \\
\hline CLABSI & $1(2.7)$ & 0 & 0 & \\
\hline NSTI & $1(2.7)$ & $4(4.9)$ & $1(11.1)$ & \\
\hline Other & 0 & $6(7.3)$ & $2(22.2)$ & \\
\hline Mortality within 28 days, n (\%) & $6(16.2)$ & $2(2.4)$ & $9(100)$ & 0.131 \\
\hline Mortality within 180 days, $\mathrm{n}(\%)$ & $14(37.8)$ & $5(6.1)$ & $9(100)$ & $<0.001$ \\
\hline
\end{tabular}

APACHE II, Acute Physiology and Chronic Health Evaluation; CCI, chronic critical illness; CLABSI, central-line associated bloodstream infection; ICU, intensive care unit; LOS, length of stay; NSTI, necrotizing soft tissue infection; RAP, rapid recovery; SD, standard deviation; SOFA, Sequential Organ Failure Assessment.

with the length of hospital stay in both the CCI and RAP groups; however, the level in the CCI group decreased more slowly and remained above $50 \mu \mathrm{g} / \mathrm{dL}$ during the 21-day observation period (Figure 1A). Serum IL-6 level in the CCI and RAP groups also declined, but in the former, the level remained above $30 \mathrm{pg} / \mathrm{mL}$ during the observation period (Figure $1 B$ ). The expression of CRP and IL-6 indicated that the CCI patients suffered persistent inflammation throughout the observation period.

Total lymphocyte count showed an upward trend in RAP patients during the observation period, whereas in CCI patients, the number increased slowly before reaching a plateau in the range of $\sim 0.75 \times 10^{9} / \mathrm{L}$ around day 7 of hospitalization (Figure 1C). Serum IL-10 level in the RAP group decreased with length of hospital stay, whereas in the CCI group, the level decreased more slowly and remained above $25 \mathrm{pg} / \mathrm{mL}$ during the observation period (Figure 1D).
Differences in total lymphocyte count and IL-10 expression between the RAP and CCI groups suggested a higher degree of immunosuppression in the CCI group.

\section{SIRT2 gene expression levels in patients}

The SIRT2 expression result show that SIRT2 expression in the CCI and RAP patients was lower than in the controls at the time of ICU admission, but increased starting on day 4 of hospitalization and continued to increase with length of hospital stay. SIRT2 expression was higher in the CCI group than in the RAP group at admission, but lower in the CCI group than in the RAP group on day 1 , and the differences were not statistically significant (Figure $2 A$ and $B$ ). SIRT2 expression was lower in the CCI group than in the RAP group on day $4(\mathrm{P}<0.01)$, day $7(\mathrm{P}<0.01)$, and day 10 $(\mathrm{P}<0.001)$ (Figure $2 C, D, E)$. However, while the level of 
A

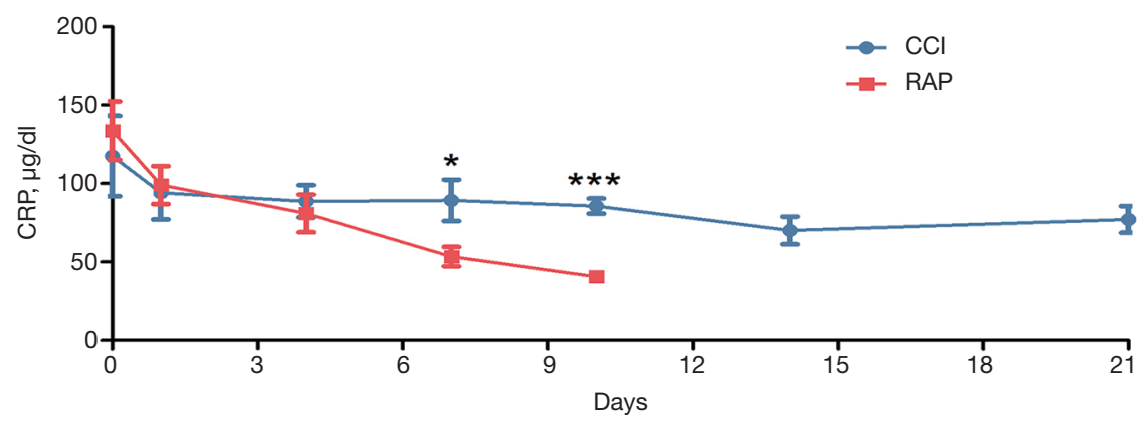

B

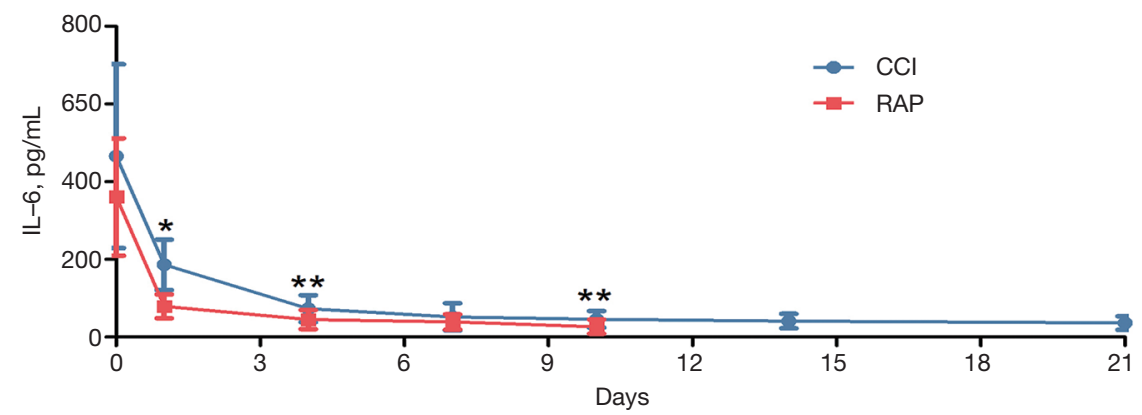

C

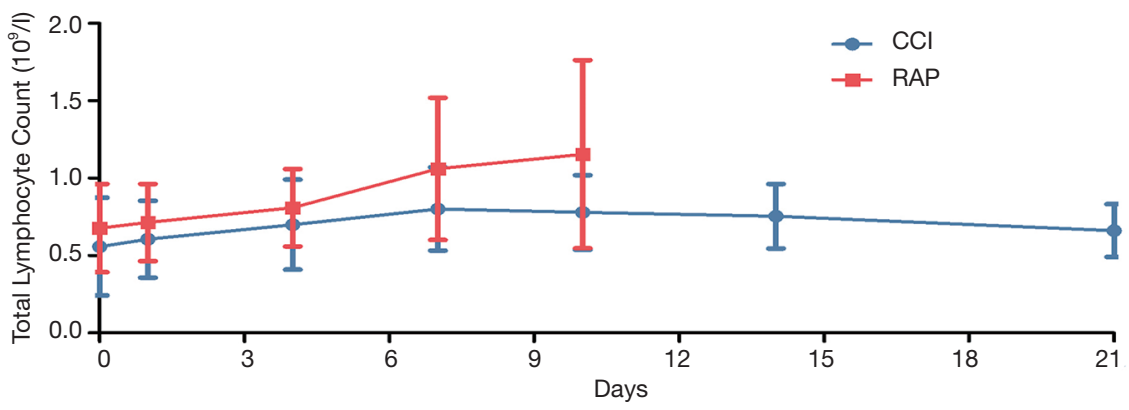

D

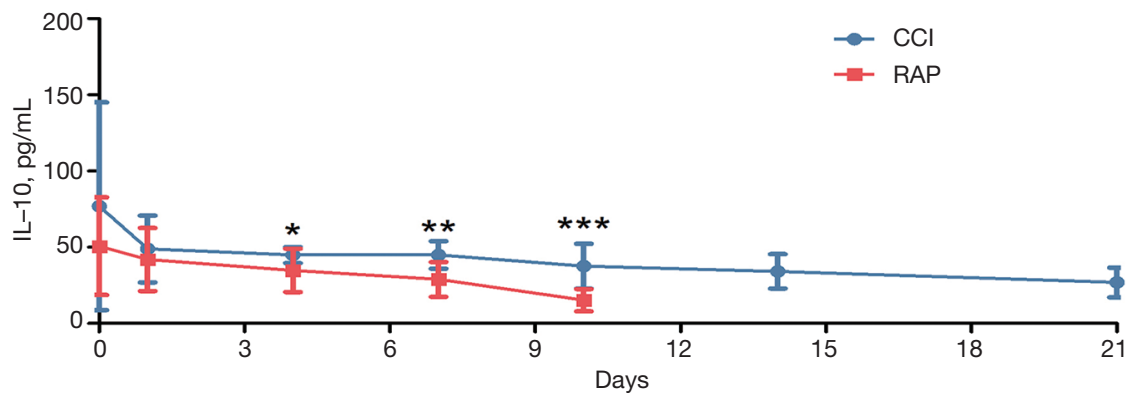

Figure 1 Markers of inflammation and immune activation in patients with sepsis or septic shock. (A) C-reactive protein (CRP) level, (B) interleukin (IL)-6 level, (C) total lymphocyte count, and (D) IL-10 level over time in patients with sepsis or septic shock who developed critical illness (CCI) or showed rapid recovery (RAP). Blue and red lines represent $\mathrm{CCI}$ and $\mathrm{RAP}$, respectively. ${ }^{*} \mathrm{P}<0.05,{ }^{* *} \mathrm{P}<0.001$, $* * * \mathrm{P}<0.0001$.

SIRT2 expression in RAP patients reached that of healthy controls by day 10, the expression level of SIRT2 in CCI patients continued to increase throughout the observation period (Figure 2F).

\section{Predictive utility of SIRT2 for CCI}

Given the difference in the temporal dynamics of SIRT2 expression between the CCI and RAP groups, we speculated that $S I R T 2$ can serve as an early marker 

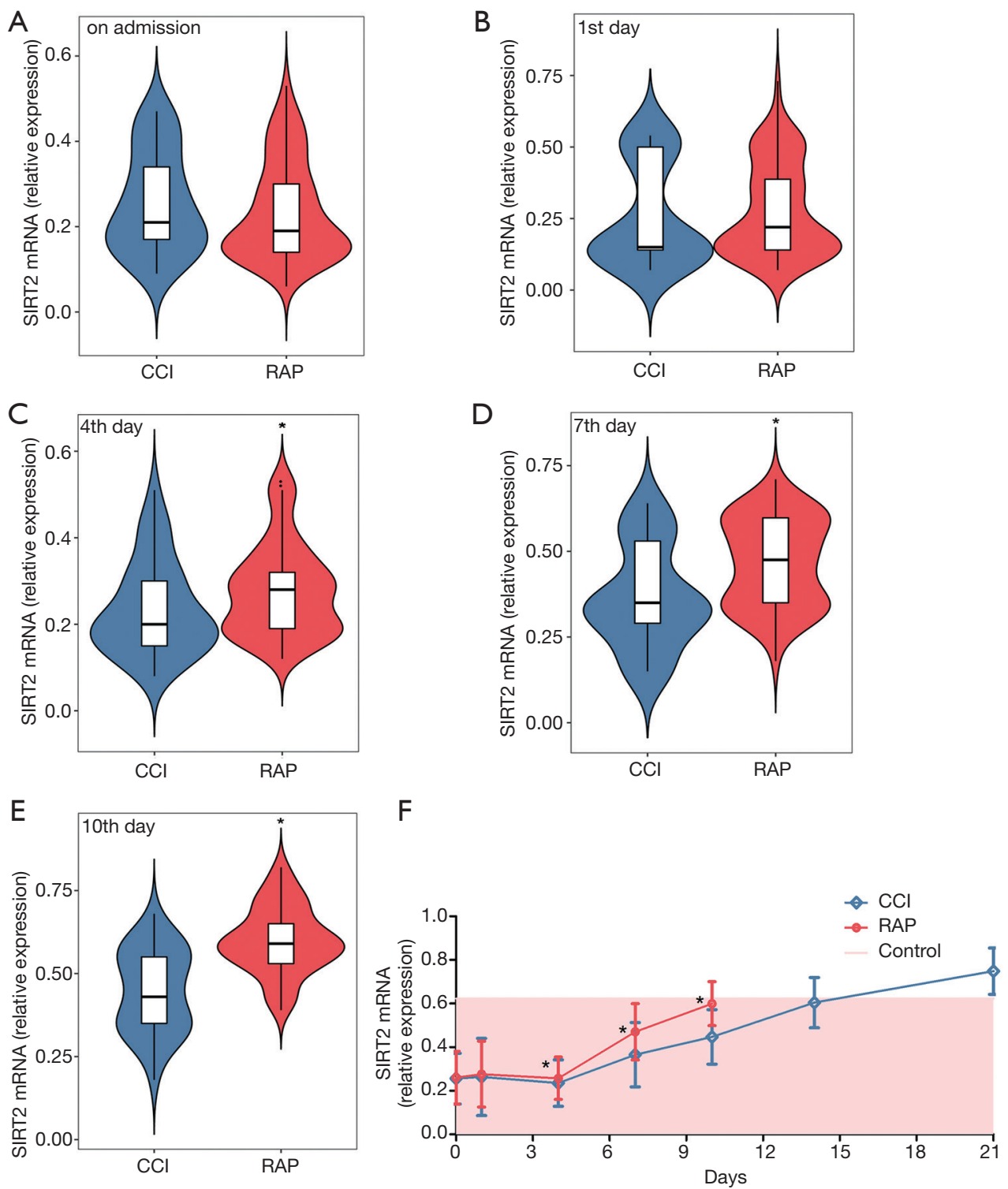

Figure 2 Sirtuin 2 (SIRT2) mRNA expression in patients with critical illness (CCI) and rapid recovery (RAP). SIRT2 transcript levels in CCI and RAP cases were evaluated at admission (A) and on day 1 (B), day 4 (C), day 7 (D), and day 10 (E) after admission. (F) Differences in SIRT2 expression levels at indicated time points in patients who develop CCI or RAP. Pink shading represents normal range. ${ }^{*} \mathrm{P}<0.05$.

for CCI in sepsis patients. To assess this possibility, we established a prediction model with APACHE II score, SOFA score, CRP, and relative SIRT2 expression level on days $1,4,7$, and 10 after enrollment as explanatory variables. Our analysis showed that the relative expression level of SIRT2 at 10 days after hospitalization could better predict the occurrence of $\mathrm{CCI}(\mathrm{P}<0.05)$. The cut-off value for SIRT2 at 10 days was 0.536 , the AUC was 0.849 [95\% confidence interval (CI): 0.747-0.921], sensitivity was 0.730 , and specificity was $0.811(\mathrm{P}<0.001)$. The cut-off value for APACHE II score at admission was 19.5, and the AUC for APACHE II score was 0.711 (95\% CI: $0.608-0.815)$. The cut-off value for SOFA score at admission was 7.5, and the AUC was 0.695 (95\% CI: 0.588-0.802) (Figure 3).

\section{Discussion}




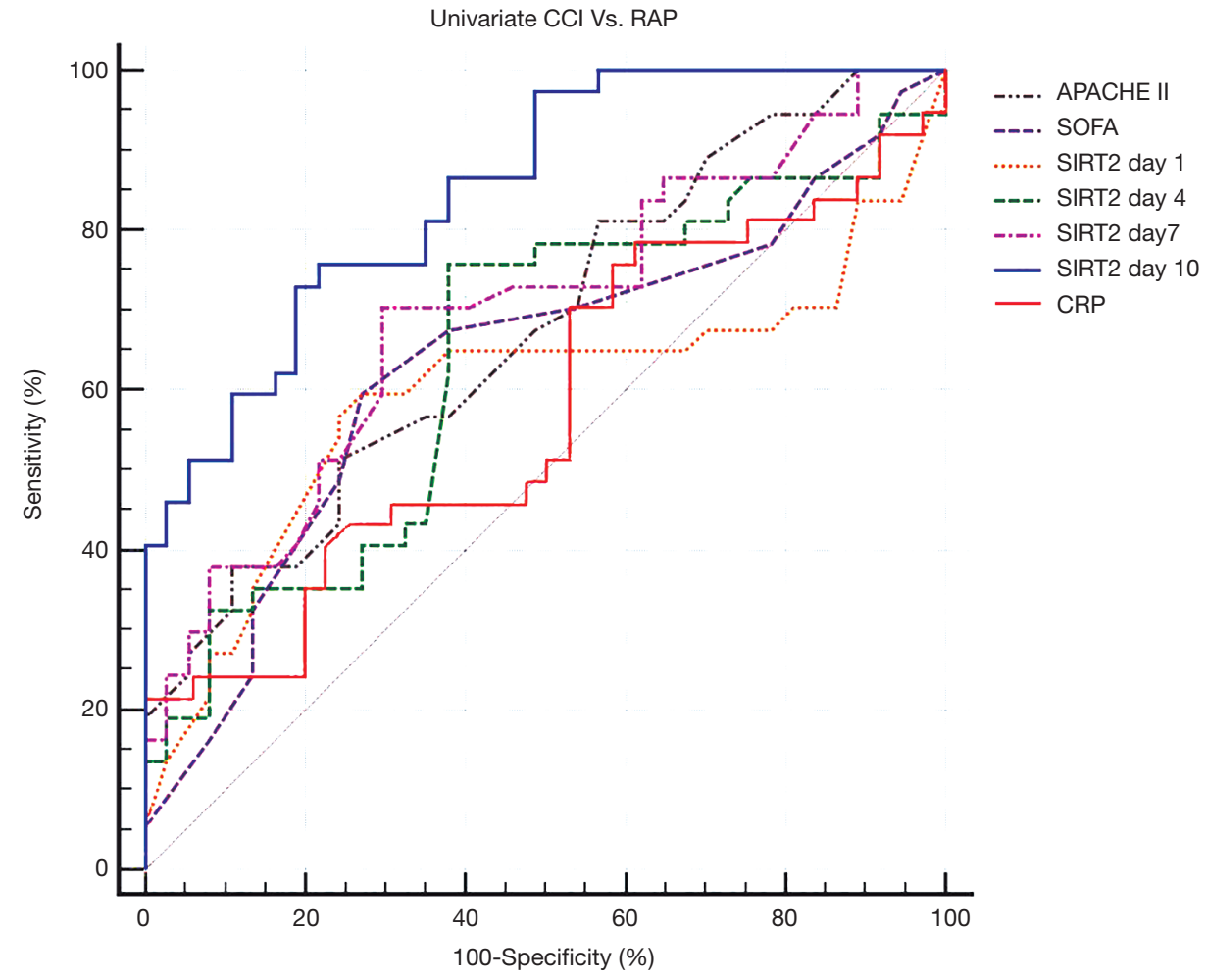

\begin{tabular}{|l|c|c|c|c|}
\hline \multicolumn{1}{|c|}{ Variable } & $n($ CCI/RAP) & AUC $(95 \% \mathrm{Cl})$ & P-value & Odds ratio (95\% CI) \\
\hline APACHE II & $119(37 / 82)$ & $0.711(0.608-0.815)$ & 0.0002 & $1.111(1.031-1.197)$ \\
\hline SOFA & $119(37 / 82)$ & $0.695(0.588-0.802)$ & 0.0007 & $1.139(1.006-1.291)$ \\
\hline CRP & $119(37 / 82)$ & $0.573(0.454-0.693)$ & 0.2015 & $1.052(1.026-1.078)$ \\
\hline SIRT2 day 1 & $119(37 / 82)$ & $0.595(0.474-0.708)$ & 0.3860 & $1.394(1.075-1.807)$ \\
\hline SIRT2 day 4 & $118(37 / 81)$ & $0.641(0.521-0.749)$ & 0.0401 & $1.000(0.993-1.009)$ \\
\hline SIRT2 day 7 & $103(37 / 66)$ & $0.696(0.579-0.798)$ & 0.0011 & $1.097(1.043-1.179)$ \\
\hline SIRT2 day 10 & $74(37 / 37)$ & $0.849(0.747-0.921)$ & 0.0001 & $1.007(0.996-1.019)$ \\
\hline
\end{tabular}

Figure 3 Receiver-operating characteristic curves for Acute Physiology and Chronic Health Evaluation (APACHE II) score, Sequential Organ Failure Assessment (SOFA) score, C-reactive protein (CRP), and sirtuin 2 (SIRT2) level for prediction of chronic critical illness (CCI) vs. rapid recovery (RAP). AUC, area under the curve; CI, confidence interval.

Improvements in the early identification, source control, and critical care management of sepsis have led to a reduction in the early mortality rate in patients $(10,11)$. However, with the increasing number of survivors, there are now more sepsis patients who progress to CCI. CCI has emerged as an epidemic among ICU survivors worldwide (9), prolonging hospital stay and increasing the cost of treatment. Moreover, CCI has increased the long-term mortality rate of sepsis patients. Since the term "chronic critical illness" was first proposed in 1985, there have been a number of reports on this phenomenon (12). There are several definitions of CCI that inform clinical diagnosis. However, CCI is generally defined as organ dysfunction persisting for more than 14 days in an ICU patient (12). The incidence of CCI after sepsis is almost $50 \%$ (13). Persistent PICS is thought to be the underlying pathophysiology in CCI (14). Patients often exhibit aberrant immunity and are observed to have 
persistent inflammation, ongoing immunosuppression, and lean muscle mass wasting associated with protein catabolism $(6,14)$. These features are major obstacles for the successful pharmacological treatment of CCI. Early detection of CCI and timely intervention to restore immune homeostasis could improve patient outcomes.

In recent research on PICS, CRP $>50 \mu \mathrm{g} / \mathrm{dL}$ and increased IL-6 and IL-8 levels accompanied by myeloidderived suppressor cell (MDSC) expansion have been considered an indicator of persistent inflammation, while a lower total lymphocyte count has been taken as evidence of immunosuppression $(6,15)$. IL-10 is an anti-inflammatory factor and important regulator of immune response (16). Studies have shown that IL-10 can increase the number of MDSCs and strengthen their immunosuppressive function (17).

SIRTs, which are part of the class III histone deacetylase family of enzymes, are a highly conserved family of proteins that were first identified in yeast $(7,18)$. There are 7 SIRTs (SIRT1-7) in this family, Recent research shows that SIRT2 is involved in inflammation in sepsis. One study reported that SIRT2 expression was downregulated in the early stage of lipopolysaccharide (LPS) stimulation (19), but showed an upward trend as the LPS stimulation time was extended (20). The expression of the SIRT2 gene was found to increase during the immune paralysis stage of a cecal ligation and perforation-induced sepsis model, and inhibiting SIRT2 expression was found to enhance microvascular inflammation (21). These findings indicate that SIRT2 expression may reflect immune response status. Several studies have reported that SIRT2 is involved in the inflammation and metabolic regulation of sepsis through its deacetylase activity (18,21-25). Research shows that SIRT2 expression was observed to decrease during hyperinflammation and increase during the hypo-inflammatory phase in a mouse model of obesity with sepsis, with direct deacetylation of nuclear factor (NF)- $\mathrm{\kappa B} / \mathrm{p} 65$ (21), while the development of CCI in sepsis patients also involves progression from the hyper-inflammatory response state of a cytokine burst to hypo-inflammation. However, the dynamics of SIRT2 gene expression in sepsis patients during progression to CCI have not been previously investigated.

In the present study, 128 patients with sepsis or septic shock were enrolled and divided into the CCI, RAP, and early death patient groups, according to their condition during hospitalization. Blood samples were collected and tested at multiple time points, and showed that the CRP concentration and the expression level of IL-6 in the CCI patients reflected the presence of persistent inflammation when compared with that of the RAP patients. Similarly, compared with the RAP group, the total lymphocyte count and expression level of IL-10 in the CCI group demonstrated immunosuppression. Importantly, SIRT2 expression was lower in patients with sepsis or septic shock than in healthy individuals. The expression of SIRT2 increased with length of hospitalization; before discharge, SIRT2 expression in RAP patients had returned to a normal range, whereas in CCI patients, the level continued to increase throughout the observation period. The predictive value of SIRT2 expression, as well as APACHE II and SOFA scores at different time points for the progression of sepsis to CCI, were analyzed. The results showed that SIRT2 transcription level on days 4, 7 and 10 can predict the occurrence of CCI, especially on day 10.

Our results showed that the expression level of SIRT2 in the RAP group returned to the normal range, while the expression level of SIRT2 in the CCI group increased slowly and remained above the normal range at the end of our observation. This may be a manifestation of the different pathophysiology of the 2 groups of patients. As mentioned earlier, SIRT2 is involved in the inflammatory response regulation of sepsis through its deacetylation effect, and the CCI patients had persistent inflammation and immunosuppression. Therefore, the characteristics of SIRT2 expression changes in CCI patients may be related to changes in patient immune homeostasis. However, its mechanism of action is unclear. The imbalance of the redox reaction caused by the excessive production of oxidants in the body and the lack of antioxidants, that is, oxidative stress, is common in patients with sepsis $(26,27)$. Studies have shown that the SIRT2 protein can be directly oxidized to reduce its deacetylation during oxidative stress, and oxidative stress can also affect the expression of SIRT family genes at the transcriptional level by regulating the binding of FOS and c-JUN heterodimer activator protein-1 to DNA $(22,28,29)$. Oxidative stress can also cause SIRT2 to remain in the cytoplasm by damaging the key protein importin-7 (IPO7) that mediates SIRT2 into the nucleus $(30,31)$. This will lead to excessive SIRT2 binding to NF- $\mathrm{KB}$ and nuclear factor-E2-related factor 2 (Nrf2) proteins in the cytoplasm of the cell, blocking NF- $\mathrm{\kappa B}$ and Nrf2 proteins from entering the nucleus through deacetylation, which not only downregulates the expression of inflammatory genes downstream of NF- $\mathrm{\kappa B}$ but also affects the expression of iron transporters downstream of $\mathrm{Nrf2}$, leading to the formation of a temporary iron pool in the cytoplasm to 
further aggravate the oxidation level of the cell $(22,32,33)$. The increase in the cell oxidation level will further induce the expression of SIRT2. This may be the mechanism by which SIRT2 expression in CCI patients increase slowly and continuously, and the mechanism by which SIRT2 expression can reflect the change in the patient's immune status. Further experimental research is required to confirm this. There are also interactions between SIRT family members, which cannot be ignored.

Our study had some limitations. First, it was conducted at a single center and had a small sample size. Second, only CRP, total lymphocyte count, IL-6, and IL-10 were used to reflect the inflammatory response status in CCI patients. Third, we only detected SIRT2 in members of the SIRT family, and did not detect SIRT family's other members. Therefore, in our future studies, we will increase the sample size and number of detection indices to validate the current findings, and further research the mechanism of SIRT2 expression changes in the progression of sepsis to CCI, as well as the possibility of SIRT2 as the therapeutic option to restore immune homeostasis for CCI.

Nonetheless, our findings indicate that the SIRT2 expression profile can be used to predict the occurrence of CCI, making it a useful marker in the clinical management of sepsis. SIRT2 may serve as a potential therapeutic target for CCI of sepsis.

\section{Acknowledgments}

Funding: This work was supported by the Science and Technology Foundation of Tianjin First Central Hospital (grant No. YCM201809) and the National Clinical Key Specially Project Foundation of the Ministry of Health in China (grant No. 2011-873).

\section{Footnote}

Reporting Checklist: The authors have completed the TRIPOD reporting checklist. Available at http://dx.doi. org/10.21037/atm-20-7975

Data Sharing Statement: Available at http://dx.doi. org/10.21037/atm-20-7975

Conflicts of Interest: All authors have completed the ICMJE uniform disclosure form (available at http://dx.doi. org/10.21037/atm-20-7975). The authors have no conflicts of interest to declare.
Ethical Statement: The authors are accountable for all aspects of the work in ensuring that questions related to the accuracy or integrity of any part of the work are appropriately investigated and resolved. The study was conducted in accordance with the Declaration of Helsinki (as revise in 2013). This observational and prospective cohort study was approved by the Tianjin First Center Hospital Ethics Committee (approval No. 2018N085KY) and informed consent was obtained from patients or their legal representatives.

Open Access Statement: This is an Open Access article distributed in accordance with the Creative Commons Attribution-NonCommercial-NoDerivs 4.0 International License (CC BY-NC-ND 4.0), which permits the noncommercial replication and distribution of the article with the strict proviso that no changes or edits are made and the original work is properly cited (including links to both the formal publication through the relevant DOI and the license). See: https://creativecommons.org/licenses/by-nc-nd/4.0/.

\section{References}

1. Singer M, Deutschman CS, Seymour CW, et al. The third international consensus definitions for sepsis and septic shock (sepsis- 3). JAMA 2016;315:801-10.

2. Rudd KE, Johnson SC, Agesa KM, et al. Global, regional, and national sepsis incidence and mortality, 1990-2017: analysis for the Global Burden of Disease Study. Lancet 2020;395:200-11.

3. Evans IVR, Phillips GS, Alpern ER, et al. Association between the New York sepsis care mandate and in-hospital mortality for pediatric sepsis. JAMA 2018;320:358-67.

4. Liu VX, Fielding-Singh V, Greene JD, et al. The timing of early antibiotics and hospital mortality in sepsis. Am J Respir Crit Care Med 2017;196:856-63.

5. Rosenthal MD, Bala T, Wang Z, et al. Chronic Critical Illness Patients Fail to Respond to Current EvidenceBased Intensive Care Nutrition Secondarily to Persistent Inflammation, Immunosuppression, and Catabolic Syndrome. JPEN J Parenter Enteral Nutr 2020;44:1237-49.

6. Hawkins RB, Raymond SL, Stortz JA, et al. Chronic Critical Illness and the Persistent Inflammation, Immunosuppression, and Catabolism Syndrome. Front Immunol 2018;9:1511.

7. Mendes KL, Lelis DF, Santos SHS. Nuclear sirtuins and inflammatory signaling pathways. Cytokine Growth Factor Rev 2017;38:98-105. 


\section{Page 10 of 10}

8. He M, Chiang HH, Luo H, et al. An Acetylation Switch of the NLRP3 Inflammasome Regulates Aging-Associated Chronic Inflammation and Insulin Resistance. Cell Metab 2020;31:580-91.e5.

9. Hawkins RB, Stortz JA, Holden DC, et al. Persistently increased cell-free DNA concentrations only modestly contribute to outcome and host response in sepsis survivors with chronic critical illness. Surgery 2020;167:646-52.

10. Horiguchi H, Loftus TJ, Hawkins RB, et al. Innate Immunity in the Persistent Inflammation, Immunosuppression, and Catabolism Syndrome and Its Implications for Therapy. Front Immunol 2018;9:595.

11. Prescott HC. Preventing Chronic Critical Illness and Rehospitalization: A Focus on Sepsis. Crit Care Clin 2018;34:501-13.

12. Rosenthal MD, Kamel AY, Rosenthal CM, et al. Chronic Critical Illness: Application of What We Know. Nutr Clin Pract 2018;33:39-45.

13. Stortz JA, Mira JC, Raymond SL, et al. Benchmarking clinical outcomes and the immunocatabolic phenotype of chronic critical illness after sepsis in surgical intensive care unit patients. J Trauma Acute Care Surg 2018;84:342-9.

14. Mira JC, Gentile LF, Mathias BJ, et al. Sepsis Pathophysiology, Chronic Critical Illness, and Persistent Inflammation- Immunosuppression and Catabolism Syndrome. Crit Care Med 2017;45:253-62.

15. Mira JC, Brakenridge SC, Moldawer LL, et al. Persistent Inflammation, Immunosuppression and Catabolism Syndrome. Crit Care Clin 2017;33:245-58.

16. Xu Y, Li Y, Liu X, et al. SPIONs enhances IL-10producing macrophages to relieve sepsis via Cav1Notch1/HES1-mediated autophagy. Int J Nanomedicine 2019;14:6779-97.

17. Bah I, Kumbhare A, Nguyen L, et al. IL-10 induces an immune repressor pathway in sepsis by promoting S100A9 nuclear localization and MDSC development. Cell Immunol 2018;332:32-8.

18. Wang X, Buechler NL, Woodruff AG, et al. Sirtuins and Immuno-Metabolism of Sepsis. Int J Mol Sci 2018;19:E2738.

19. Lin J, Sun B, Jiang C, et al. Sirt2 suppresses inflammatory responses in collagen-induced arthritis. Biochem Biophys Res Commun 2013;441:897-903.

20. Pais TF, Szegő ÉM, Marques O, et al. The NADdependent deacetylase sirtuin 2 is a suppressor of microglial activation and brain inflammation. EMBO J 2013;32:2603-16.

21. Wang X, Buechler NL, Martin A, et al. Sirtuin-2
Xu et al. SIRT2 expression during sepsis to chronic critical illness

Regulates Sepsis Inflammation in ob/ob Mice. PLoS One 2016;11:e0160431.

22. Wang X, Buechler NL, Long DL, et al. Cysteine thiol oxidation on SIRT2 regulates inflammation in obese mice with sepsis. Inflammation 2019;42:156-69.

23. Lee AS, Jung YJ, Kim D, et al. Sirt2 ameliorates lipopolysaccharide-induced inflammation in macrophages. Biochem Biophys Res Commun 2014;450:1363-9.

24. Buechler N, Wang X, Yoza BK, et al. Sirtuin 2 regulates microvascular inflammation during sepsis. J Immunol Res 2017;2017:2648946.

25. Ciarlo E, Heinonen T, Theroude C, et al. Sirtuin 2 deficiency increases bacterial phagocytosis by macrophages and protects from chronic staphylococcal infection. Front Immunol 2017;8:1037.

26. Sies H. Oxidative stress: a concept in redox biology and medicine. Redox Biol 2015;4:180-3.

27. Mantzarlis K, Tsolaki V, Zakynthinos E. Role of Oxidative Stress and Mitochondrial Dysfunction in Sepsis and Potential Therapies. Oxid Med Cell Longev 2017;2017:5985209.

28. Sies H, Berndt C, Jones DP. Oxidative Stress. Annu Rev Biochem 2017;86:715-48.

29. Min L, Ji Y, Bakiri L, et al. Liver cancer initiation is controlled by AP-1 through SIRT6-dependent inhibition of survivin. Nat Cell Biol 2012;14:1203-11.

30. Hakim A, Barnes PJ, Adcock IM, et al. Importin-7 mediates glucocorticoid receptor nuclear import and is impaired by oxidative stress, leading to glucocorticoid insensitivity. FASEB J 2013;27:4510-9.

31. Eldridge MJG, Pereira JM, Impens F, et al. Active nuclear import of the deacetylase Sirtuin-2 is controlled by its C-terminus and importins. Sci Rep 2020;10:2034.

32. Yang X, Park SH, Chang HC, et al. Sirtuin 2 regulates cellular iron homeostasis via deacetylation of transcription factor NRF2. J Clin Invest 2017;127:1505-16.

33. Li J, Cao F, Yin HL, et al. Ferroptosis: past, present and future. Cell Death Dis 2020;11:88.

(English Language Editor: R. Scott)

Cite this article as: $\mathrm{Xu} \mathrm{H,} \mathrm{Li} \mathrm{J,} \mathrm{Yu} \mathrm{X,} \mathrm{Zhang} \mathrm{H,} \mathrm{Gao} \mathrm{H,}$ Wang B, Wang Y. Sirtuin 2 expression levels may predict the progression of sepsis survivors to chronic critical illness. Ann Transl Med 2021;9(2):150. doi: 10.21037/atm-20-7975 